\title{
Ky Fan's inequality with binomial expansion
}

\author{
Jamal Rooin \\ Jamal Rooin received his M.S. from the Teacher Education University in Teheran, \\ Iran, in 1989. In 1997 he became a Ph.D. student at that university, where he graduated \\ in the year 2002. He is now assistant professor at the Institute for Advanced Studies in \\ Basic Sciences in Zanjan, Iran. His mathematical research interests include the theory \\ of inequalities, problems in analysis and functional analysis.
}

In this note, given $n$ arbitrary nonnegative real numbers $x_{1}, \ldots, x_{n}$, we denote by $A_{n}$ and $G_{n}$, the unweighted arithmetic and geometric means of $x_{1}, \ldots, x_{n}$, respectively, i.e.,

$$
A_{n}=\frac{1}{n} \sum_{i=1}^{n} x_{i}, \quad G_{n}=\prod_{i=1}^{n} x_{i}^{1 / n},
$$

and moreover, if $x_{i} \in(0,1 / 2]$, we denote by $A_{n}^{\prime}$ and $G_{n}^{\prime}$, the unweighted arithmetic and geometric means of $1-x_{1}, \ldots, 1-x_{n}$, respectively, i.e.,

$$
A_{n}^{\prime}=\frac{1}{n} \sum_{i=1}^{n}\left(1-x_{i}\right), \quad G_{n}^{\prime}=\prod_{i=1}^{n}\left(1-x_{i}\right)^{1 / n} .
$$

In 1961 the following remarkable inequality, due to Ky Fan, was published for the first time in the well-known book Inequalities by Beckenbach and Bellman [2, p. 5]:

If $x_{i} \in(0,1 / 2]$, then

$$
\frac{A_{n}^{\prime}}{G_{n}^{\prime}} \leq \frac{A_{n}}{G_{n}}
$$

with equality holding if and only if $x_{1}=\cdots=x_{n}$.

Die Ungleichung von Ky Fan ist 1961 in der bekannten Enzyklopädie von Beckenbach und Bellman zum ersten Mal publiziert worden. Ausgangspunkt ist die Ungleichung zwischen dem arithmetischen und dem geometrischen Mittel, kurz AGM-Ungleichung. Die Ungleichung von Ky Fan besagt, dass sich die Diskrepanz zwischen dem arithmetischen und dem geometrischen Mittel verkleinert, wenn die gegebenen Zahlen $x_{k} \in\left(0, \frac{1}{2}\right]$ durch $x_{k}^{\prime}:=1-x_{k}$ ersetzt werden. In der vorliegenden Note wird ein weiterer, besonders einfacher Beweis dieser Ungleichung gegeben. 
Inequality (1) has evoked the interest of several mathematicians and in numerous articles new proofs, extensions, refinements and various related results have been published; see the survey paper [1] and the references therein.

By replacing the apparent binomial expansion, it is easily established that the following recursive identity holds (see [3]):

$$
A_{n}-G_{n}=\frac{1}{n} \sum_{k=2}^{n}\left(\begin{array}{l}
n \\
k
\end{array}\right) A_{n-1}^{\frac{n-k}{n}}\left(x_{n}^{1 / n}-A_{n-1}^{1 / n}\right)^{k}+x_{n}^{1 / n}\left(A_{n-1}^{\frac{n-1}{n}}-G_{n-1}^{\frac{n-1}{n}}\right) .
$$

Using this identity, we now give a striking new proof of (1) which shows the power of binomial expansion on the one hand and the close relations between AGM and Ky Fan inequalities on the other:

Actually, dividing each side of (2) by

$$
G_{n}=G_{n-1}^{\frac{n-1}{n}} x_{n}^{\frac{1}{n}} \neq 0
$$

we get the following recursive identity

$$
\frac{A_{n}}{G_{n}}=\frac{1}{n} \sum_{k=2}^{n}\left(\begin{array}{l}
n \\
k
\end{array}\right)\left(\frac{A_{n-1}}{G_{n-1}}\right)^{\frac{n-k}{n}} \frac{\left(x_{n}^{\frac{1}{n}}-A_{n-1}^{\frac{1}{n}}\right)^{k}}{G_{n-1}^{\frac{k-1}{n}} x_{n}^{\frac{1}{n}}}+\left(\frac{A_{n-1}}{G_{n-1}}\right)^{\frac{n-1}{n}} .
$$

Now, we proceed by induction on $n$. If $n=1$, there is nothing to prove. Suppose that $n \geq 2$ and (1) holds for $n-1$. If $x_{1}=\cdots=x_{n}$, then obviously equality holds in (1). Let $x_{i}$ 's not all be equal. By a rearrangement if necessary, we can suppose that $x_{n}=\max _{1 \leq i \leq n} x_{i}$. Now, considering

$$
\frac{A_{n}^{\prime}}{G_{n}^{\prime}}=\frac{1}{n} \sum_{k=2}^{n}\left(\begin{array}{l}
n \\
k
\end{array}\right)\left(\frac{A_{n-1}^{\prime}}{G_{n-1}^{\prime}}\right)^{\frac{n-k}{n}} \frac{\left(\left(1-x_{n}\right)^{\frac{1}{n}}-A_{n-1}^{\prime \frac{1}{n}}\right)^{k}}{G_{n-1}^{\prime \frac{k-1}{n}}\left(1-x_{n}\right)^{\frac{1}{n}}}+\left(\frac{A_{n-1}^{\prime}}{G_{n-1}^{\prime}}\right)^{\frac{n-1}{n}}
$$

with the induction hypothesis

and taking into account that

$$
\frac{A_{n-1}}{G_{n-1}} \geq \frac{A_{n-1}^{\prime}}{G_{n-1}^{\prime}}
$$

$$
\begin{gathered}
G_{n-1}<G_{n-1}^{\prime}, \quad A_{n-1}<A_{n-1}^{\prime}, \quad x_{n} \leq 1-x_{n}, \\
A_{n-1}^{\prime}=1-A_{n-1}, \quad x_{n}>A_{n-1},
\end{gathered}
$$

and

$$
\begin{aligned}
x_{n}^{\frac{1}{n}}-A_{n-1}^{\frac{1}{n}} & =\frac{x_{n}-A_{n-1}}{\sum_{i=0}^{n-1} x_{n}^{\frac{i}{n}} A_{n-1}^{\frac{n-1-i}{n}}}>\frac{x_{n}-A_{n-1}}{\sum_{i=0}^{n-1}\left(1-x_{n}\right)^{\frac{i}{n}} A^{\prime}{ }_{n-1}^{\frac{n-1-i}{n}}} \\
& =\left|\left(1-x_{n}\right)^{\frac{1}{n}}-A_{n-1}^{\prime \frac{1}{n}}\right|,
\end{aligned}
$$

we get $\frac{A_{n}}{G_{n}}>\frac{A_{n}^{\prime}}{G_{n}^{\prime}}$ and the proof is completed. 


\section{References}

[1] Alzer, H.: The inequality of Ky Fan and related results. Acta Appl. Math. 38 (1995), 305-354.

[2] Beckenbach, E.F.; Bellman, R.: Inequalities. Springer-Verlag, Berlin 1961.

[3] Rooin, J.: AGM inequality with binomial expansion. Elem. Math. 58 (2003), 115-117.

\section{Jamal Rooin}

Department of Mathematics

Institute for Advanced

Studies in Basic Sciences

Zanjan, Iran

e-mail:rooin@iasbs.ac.ir 